\title{
Origins of Deviations from Transition-State Theory: Formulating a New Kinetic Rate Law for Dissolution of Silicates
}

(Project Number: 81908)

\section{Principal Investigator}

\author{
Jonathan Icenhower \\ Pacific Northwest National Laboratory \\ P.O. Box 999, MSIN K6-81 \\ Richland, WA 99352 \\ 509-372-0078 (phone) \\ 509-376-2210 (fax) \\ jonathan.icenhower@pnl.gov
}

\section{Co-Investigators}

B. P. McGrail

Pacific Northwest National Laboratory

P.O. Box 999, MSIN K6-81

Richland, WA 99352

509-376-9193 (phone)

509-376-2210 (fax)

pete.mcgrail@pnl.gov

\author{
A. Luttge \\ Rice University \\ Dept. Geology and Geophysics-MS 126 \\ P.O. Box 1892 \\ Houston, TX 77251-1892 \\ 713-348-6304 (phone) \\ aluttge@ rice.edu
}

D. London University of Oklahoma Dept. Geology and Geophysics 100 E. Boyd Str. 810 SEC Norman, OK 73019-0628 405-325-7626 (phone)

dlondon@ou.edu 


\section{Research Objective}

Present models for dissolution of silicate minerals and glasses, based on transition-state theory (TST), overestimate the reaction rate as solution compositions approach saturation with respect to the rate-governing solid. Therefore, the reactivity of key materials in the environment, such as feldspar, mica, and borosilicate glass, is uncertain, and any prediction of future aqueous durability is suspect. The core objective of this investigation is to determine the origin of these discrepancies and to fashion a quantitative model that reliably predicts the reactivity of silicate materials in realistic environmental conditions. This is being accomplished using newly developed experimental techniques checked against computer simulations based upon firstprinciple theory.

\section{Research Progress and Implications}

Research thus far has focused upon select borosilicate glass compositions and feldspar studies. Dissolution experiments with borosilicate glass compositions along the sodium silicatereedmergnerite join are aimed at revealing the relationship between melt (glass) structure and durability. Glasses with increasing amounts of sodium silicate contain a larger fraction of nonbridging oxygen (NBO) compared to end-member reedmergnerite (the boron version of albite). We selected this series of glasses because a number of models advocate a relationship between the number of NBOs and durability. Another glass series under investigation is a set of sodium nepheline compositions with variable amounts of Al/B. The glass compositions are designed such that the amount of "excess" sodium (i.e., more sodium than $\mathrm{Al}+\mathrm{B}$ on a molar basis) is varied. These glass compositions probe the effects of $\mathrm{Na}-\mathrm{H}$ exchange, identified as a potential factor in the dissolution of certain glass compositions at rates different from those expected from TST models. Another facet of this investigation is to use the data gleaned from the new technique of vertical scanning interferometery (VSI) to quantify the dissolution of glass and feldspar as the solution approaches saturation. These latter data have been used to construct a new rate model for the dissolution kinetics of crystalline solids.

\section{Durability Experiments with Borosilicate Glass}

Single-pass flow-through (SPFT) experiments have been used to quantify rates of reaction in the glass specimens. Our investigations have revealed that the forward rate of dissolution is constant with respect to glass chemical composition. This observation is consistent with the notion that glass dissolution is rate-limited by the rupture of the $\mathrm{Si}-\mathrm{O}$ bond and is insensitive to even gross differences in major and minor element composition. This is a radical departure from the received wisdom of glass dissolution kinetics and must be carefully checked for its veracity. Ion-exchange kinetics appears to decrease in importance as molar $\mathrm{Na} /(\mathrm{Al}+\mathrm{B})$ approaches unity. Again, this is consistent with our theory and implies that controlling the exchange of $\mathrm{Na}^{+}$for $\mathrm{H}^{+}$ 
during reaction will strongly ameliorate the matrix dissolution rate. The $\mathrm{Na}-\mathrm{H}$ exchange leaves behind $\mathrm{OH}^{-}$, which raises the local $\mathrm{pH}$ in the vicinity of the $\mathrm{Si}-\mathrm{O}$ bond and catalyses the silanation reaction, and, ultimately, the release of $\mathrm{H}_{4} \mathrm{SiO}_{4}$ to solution, thereby affecting the matrix dissolution rate.

\section{Vertical Scanning Interferometry Experiments}

Dissolution rates of silicate materials also have been quantified using VSI experiments at Rice University. By measuring the absolute height difference between a reacted and a pristine surface, the dissolution rate of a glass monolith or crystal can be determined without relying on solution analyses. This is important because obtaining measurable quantities of elements from a dissolving silicate near saturation, using SPFT techniques, is very difficult. Our experiments have revealed a viable mechanism for the dissolution of silicate crystals based on step waves emanating from etch pits. Etch pits are the result of dislocations (crystal imperfections) that intersect the mineral surface. The step waves move across the surface of the mineral, producing a relatively flat surface punctuated by the etch pits, as revealed by VSI techniques. We predict that the movement of the step waves will slow when dissolution cavities form as the system nears saturation. This theory also can explain the nonlinearity in the dissolution rates as the system approaches saturation, although more experiments are needed to confirm this.

\section{Synthesis Experiments}

Experiments on methods to grow synthetic feldspar and micas are being conducted at the University of Oklahoma. Once the methods are perfected, researchers will work to synthesize crystals doped with isotopic tracers for use in dissolution experiments near saturation.

\section{Planned Activities}

We will complete glass-water reaction experiments with borosilicate glass compositions along the feldspar-jadeite-nepheline join. In all of these experiments, substantial amounts of Al will be substituted for boron, making the glass compositions relevant to the needs of the Hanford Site. All of these experiments will be conducted near saturation and will aid us in quantifying the deviation from model expectations.

The dissolution rate of select glass coupons will be determined using VSI techniques. These experiments will be conducted in June 2002 and are the first study of their kind. Because no relevant studies exist to guide us, we are unsure of what surface features may be revealed as dissolution proceeds (although it is likely that etch pits will not be a concern). However, the method will be useful in calibrating the difference, if any, between rates obtained from VSI and SPFT methods. 
Dissolution measurements using the VSI methods will be completed on feldspar specimens near saturation. These will be compared against rate measurements obtained from feldspar crystals containing radiotracers.

We will refine the method for synthesizing feldspar crystals. Once completed, the method will be used to grow radiotracer-containing crystals at Pacific Northwest National Laboratory for dissolution work.

\section{Information Access}

Icenhower JP, BP McGrail, A Luttge, and D London. Dissolution Behavior of Aluminoborosilicate Glass Near Silica Saturation: Origin of Deviation from TST Expectations. Invited talk for EMSL 2002, Symposia and User's Meeting, May 22, 2002, Richland, WA.

Lasaga AC and A Luttge. Mineralogical Approaches to Fundamental Crystal Dissolution Kinetics-I. Submitted to American Mineralogist. 